\title{
LA FILOSOFÍA DE LA RELIGIÓN NATURAL Y DEL ARTE EN LA FENOMENOLOGÍA DEL ESPÍRITU DE HEGEL
}

\author{
THE PHILOSOPHY OF NATURAL AND ART RELIGION IN THE \\ HEGEL'S PHENOMENOLOGY OF SPIRIT
}

Ángel Gómez Navarro*

\begin{abstract}
RESUMEN
En el presente escrito se describe la filosofía de la religión natural y del arte, en el despliegue de sus diversas figuras, tal como ha sido desarrollada por Hegel en su Fenomenología del Espíritu, teniendo en cuenta los comentarios de dos filósofos especialistas: Jean Hyppolite y Ramón Valls Plana. El propósito consiste en conocer cómo Hegel no sólo incorpora a las religiones no reveladas en su sistema filosófico y reconstruye su historia, sino que también plantea el problema de su respectiva verdad.
\end{abstract}

\section{PALABRAS CLAVE}

Filosofía, religión, fenomenología, conciencia, espíritu, manifestación

\begin{abstract}
ABSTRAC
This paper describes the philosophy of natural religion and art, in the deployment of various figures, as it has been developed by Hegel in his Phenomenology of Spirit, taking into account the comments of two philosophers specialists: Jean Hyppolite y Ramón Valls Plana. The purpose is to know how Hegel, not only incorporated to unrevealed religions in his system philosophy and reconstructs its history but also pose the problem of their respective truth.
\end{abstract}

\section{KEY WORDS}

Philosophy, religion, phenomenology, consciousness, spirit, manifestation

* Universidad Femenina del Sagrado Corazón: agomez@unife.edu.pe 


\section{Introducción}

Las filosofías alemanas de la religión surgidas en el siglo XIX tienen como grandes protagonistas a Georg Wilhelm Hegel y a Friedrich Schleiermacher. Tales filosofías fueron absolutamente inconciliables porque cada uno representaba posiciones y métodos filosóficos antagónicos. El primero, con la mediación del 'concepto' y, el segundo, con la inmediatez del 'sentimiento'. Sin embargo, los dos filósofos tratan de responder a la crisis de la religión protagonizada por el hombre moderno que se inició con la crítica de la ilustración, y por lo mismo buscaron promover una vida reconciliada y plenamente humana.

En este escrito nos limitaremos a plantear una descripción general de la filosofía de la religión natural y del arte, en el despliegue de las diversas figuras, tal como ha sido desarrollada por Hegel en su Fenomenología del Espíritu, publicada por vez primera en 1807, teniendo en cuenta los comentarios de dos filósofos especialistas: Jean Hyppolite (1974) y Ramón Valls Plana (1994). El propósito consiste en conocer cómo Hegel no sólo incorpora en el despliegue del espíritu a tales religiones no reveladas y reconstruye su historia, sino que también plantea el problema de su respectiva verdad.

Partimos citando el siguiente párrafo del Hegel maduro con el que inicia sus famosas lecciones sobre la religión, en la Berlín de 1821: "Nuestro objeto es lo simplemente verdadero, la misma verdad, la zona en la que se resuelven todos los enigmas del mundo, todas las contradicciones del pensamiento que escruta a fondo todos los dolores del sentimiento; la región de la verdad eterna y de la eterna quietud, la misma verdad absoluta, la satisfacción absoluta. Aquello por lo que el hombre es hombre y se distingue del animal es la conciencia, el pensamiento en general y más concretamente: que el hombre es espíritu. El centro del espíritu se despliega en múltiples formas y todas las distintas ciencias que irradian de él, las artes y la trama infinita de relaciones entre los hombres, los intereses de la vida política, usos y costumbres, actividades e historicidad, goce y todo cuanto tiene valor para nosotros $\mathrm{y}$ merece consideración; lo que nos da honor y satisfacción; todo aquello en que el hombre busca su destino; sus virtudes y su felicidad; aquello de donde el arte y la ciencia obtienen orgullo y fama; las relaciones que conducen a sus libertades y su voluntad: todo esto halla su último centro en la religión, en el único pensamiento, en la conciencia, en el sentimiento de Dios, que es el punto de partida y de llegada de todo, de quien todo toma principio y al que todo vuelve... Puesto que Dios es el principio y el termino de todo obrar, comenzar y querer; por ende, todos los hombres y pueblos tienen conciencia de Dios, de su sustancia absoluta como verdad que es la verdad de sí mismo".

En este texto, Hegel sitúa la filosofía de la religión en función de su 'sistema' filosófico, con la pretensión de mostrar su centralidad poniendo de manifiesto la convicción de que en la religión convergen todas las ciencias y las artes, y cómo estas quedan supeditadas a la determinación definitiva que le ha sido reservada a la

1 La filosofía de la religión de Schleiermacher presenta de un modo muy novedoso el significado y sentido de la religión, pues la concibe como un puro sentimiento de dependencia hacia lo Infinito que sólo puede ser captado por medio de la intuición. Cf. Schleiermacher, F. (1990) Discurso sobre la religión. Madrid: Tecnos. 
filosofía en la cual Dios aparece como el espíritu absoluto, la sustancia y verdad única, en quien se resuelve y disuelve todo cuanto se examina.

\section{La Religión en la Fenomenología del Espíritu ${ }^{2}$}

Hegel estudia la religión en las diversas figuras en que esta se manifiesta, tales como la conciencia, la autoconciencia, la razón y el espíritu, pero solamente en modo general y desde el punto de vista de la conciencia, pues para él, "esta no es la esencia absoluta en sí y para sí misma, no es la autoconciencia del espíritu la que se ha manifestado en esas figuras" (Hegel, 395).

La diferencia que hay entre la conciencia y la autoconciencia lo explica el filósofo francés, Jean Hyppolite, afirmando que "el espíritu se sabe a sí mismo espíritu, pero la conciencia por medio de la cual se representa a sí misma como objeto resulta inadecuada para este saber absoluto de sí mismo y debe progresar hasta que dicho objeto se haya convertido en la figura del espíritu mismo que se sabe como espíritu. Como espíritu del mundo, espíritu efectivo, el citado objeto no se ha reconciliado todavía con su esencia, con el espíritu infinito" (Hypolitte, 487).

Hegel dirá que "en la medida en que el espíritu se representa en la religión a él mismo, es ciertamente conciencia y la realidad encerrada en la religión es la figura y el ropaje de su representación. Pero la realidad no experimenta de nuevo en esta representación su pleno derecho, a saber, el de no ser solamente ropaje, sino ser allí independiente y libre; y a la inversa, al faltarle la perfección en ella misma, la religión es una figura determinada que no alcanza aquello que debe presentar, o sea el espíritu consciente de sí mismo. Para poder expresar el espíritu consciente de sí, su figura no debiera ser otra cosa que él y debiera manifestarse o ser real como es en su esencia" (Hegel, 397).

Es por esto que la forma suprema de la religión es la religión revelada, porque en ella el espíritu se da a sí mismo como es en su esencia, ya que como dice Hyppolite, la encarnación efectiva de Dios, su muerte y su resurrección en la comunidad será el propio ser ahí del espíritu que se sabe tal como es. Y en ese preciso momento el espíritu del mundo o el espíritu finito, se habrá reconciliado con el espíritu infinito; dicha reconciliación, precisamente, se convertirá en el objeto de la conciencia. Al final, el espíritu efectivo debe hacerse idéntico al espíritu absoluto, y el espíritu absoluto debe saberse en el espíritu efectivo como espíritu absoluto 3 .

En efecto, para Hegel las religiones son un fenómeno del espíritu en camino hacia el saber absoluto, y por ello, como afirma Valls Plana, la religión es la "autoconciencia del espíritu" bajo la forma de representación. [En efecto, la religión todavía no es el saber absoluto, pero sí le precede]. Las demás religiones son formas inferiores y previas que preparan y esbozan lo que será el cristianismo al que Hegel presenta como la religión revelada absoluta.

2 En esta obra, Hegel trata de la manifestación del Espíritu, es decir, de la autoconciencia de sí siguiendo el método filosófico de la dialéctica, que parte de lo concreto hasta llegar a la autoconciencia de sí y para sí, en donde el Espíritu sigue un proceso de desarrollo o despliegue en tres momentos: referencialidad, diferenciación e identidad.

3 Hyppolite señala que al final de la dialéctica de la religión ya no habrá superación, pues el espíritu en el mundo será el mismo espíritu absoluto, no habrá simbolismo ni trascendencia. Lo que el espíritu va contemplar en su historia es el mismo en su auténtica figura. Todo será revelado. Sin embargo, incluso en la religión revelada hay todavía una cierta superación que solo se produce en el saber absoluto. 
Esta dialéctica filosófica del espíritu se puede apreciar ya en la misma estructura de la división del capítulo referida a la religión, la cual está estructurada según los momentos generales del espíritu: La sección A, dedicada a la religión natural, corresponde al momento de "conciencia". La sección B, titulada la religión del arte, representa el momento de "autoconciencia". La sección C, o religión revelada, representa la "unidad de ambas", es decir, el momento llamado "razón". Sin embargo, como se ha señalado, aquí sólo le dedicaremos espacio a la religión natural y del arte, sin dejar de mencionar algo de la verdad que Hegel le asigna a la religión revelada.

\section{A. La religión natural}

A pesar de que la religión es una, las religiones se distinguen; y puesto que es autoconciencia bajo la forma de conciencia, es ulteriormente determinable. La determinación concreta depende de la forma o figura que en cada caso tome la conciencia. Así, parafraseando a Valls Plana tenemos que, la primera figura religiosa (la religión de la esencia luminosa), corresponde a la conciencia determinada como conciencia sensible; la segunda figura religiosa (la religión de la planta y el animal) está determinada por una conciencia percipiente y; por último, la religión en su tercera figura (religión del artesano) corresponderá a una conciencia bajo la forma de entendimiento.

[Tales figuras religiosas son paralelas a las tres figuras de la conciencia estudiadas en los tres primeros capítulos de la fenomenología| ${ }^{4}$. Y cada una de las religiones contiene los elementos de las demás. De ahí que para Hegel la distinción entre las religiones consiste solamente en que cada una de ellas destaca o acentúa uno de esos elementos. Ese elemento se constituye en "principio" de aquella religión como representación "propia" de la esencia. Esa representación, sin embargo, a pesar de su forma "objetiva" está superada en el seno de la religión misma, porque la religión mantiene ese elemento en "el elemento del pensamiento". Según esto, y siguiendo a Valls Plana, cada religión es consciente de los límites de su representación del absoluto, sabe que es una representación objetiva de algo que está más allá de los objetos de experiencia. En otras palabras, la religión, siendo autoconciencia representada, mantiene siempre un mayor peso específico de la autoconciencia sobre la representación.

\section{a) La esencia luminosa}

La primera representación religiosa concibe a la divinidad como luz. Según Valls Plana esta figura correspondería a la religión persa porque está emparentada con la conciencia sensible (primera figura de la Fenomenología) y también con el comienzo de la autoconciencia, o sea con la dialéctica del señorío y servidumbre. En esta primera figura religiosa, la más inmediata, es extrema la separación entre divinidad y mundo, separación que deberá reducirse hasta llegar a la identidad en la religión absoluta. De ahí que Valls Plana comente que esta primera figura es una figura menos configurada: "figura de la carencia de figura", el primer despliegue

4 Siguiendo la lógica de la Fenomenología del Espíritu, la religión natural corresponde a la conciencia, pues aquí el espíritu se aparece en ella con la forma del ser inmediato; la religión del arte corresponde a la autoconciencia, ya que el espíritu se manifiesta en ella con la forma de las obras de arte; la religión revelada corresponde a la razón, porque el espíritu se aparece en ella como en sí y para sí mismo. Al mismo tiempo, cada uno de estos momentos coincide con un espíritu real en la historia (Hyppolite, 492). 
auroral del secreto del espíritu que es autoconciencia. Este secreto debe manifestarse porque es la necesidad misma, el origen de todo despliegue que debe determinarse cada vez más hasta alcanzar la determinación que es el origen de todas las manifestaciones: la autoconciencia.

\section{b) La planta y el animal}

Esta segunda figura de la religión natural corresponde a las religiones de la India primitiva, al culto a las plantas y a los animales. Esta figura religiosa está emparentada con la percepción de la cosa sensible. Aparece aquí en la divinidad una pluralidad de atributos cuya primera representación nos lo ofrece la variedad del mundo vegetal: "El espíritu se escinde en la innumerable pluralidad de espíritus más débiles y más fuertes, más ricos y más pobres" (Hegel, 404).

Hegel utiliza aquí el término "panteísmo" para designar esa forma de religiosidad porque para él ese término está ligado a la representación tomada del mundo natural. La religión de la planta mantiene quietas las diferencias. La vida vegetal presenta una variedad infinita de formas que coexisten pacíficamente. Hegel ve en esta representación religiosa una "representación del sí mismo sin sí mismo". Pero la religión de la planta pasa en seguida a la religión del animal, y en ella se representa ya la oposición entre las distintas especies en que la vida se divide. En la religión del animal, por tanto, se avanza en la representación de la mismidad. Aquí, Valls Plana afirma que, para Hegel, esta religión del animal corresponde a los pueblos guerreros que luchan entre sí oponiendo sus particularidades (mostrando así una correlación entre religiosidad y vida política). Sin embargo, la actividad negativa de la mismidad no es, en sí misma una fuerza simplemente destructora, sino que en ella el sujeto se produce consumiéndose.

\section{c) El artesano}

Esta es la tercera y última figura de la religión natural. Corresponde a la religión egipcia y al tercer grado de la conciencia objetiva denominada: "Fuerza y Entendimiento". En esta religión la divinidad se presenta como artesano productor; pero todavía es una "una actividad condicionada y la formación de algo". Es un trabajo instintivo que no capta el pensamiento de la mismidad y no brota de ese pensamiento. La primera obra objetiva de esta religión son los obeliscos y pirámides, figuras geométricas lineales, rectas sin curvas inconmensurables.

Esta representación religiosa, al acercar el rayo de sol a los colosos, empieza a acercar alma y cuerpo y empieza a buscar una representación sintética. El que trabaja debe formar cuerpo con su obra; pero esta exigencia se cumplirá en el estadio superior, en la religión del arte propia de los pueblos helénicos.

Respecto a la arquitectura de los templos egipcios, las columnas están construidas básicamente de formas vegetales que recuerdan a las plantas; pero la forma natural está ya dominada por el entendimiento y esa arquitectura, a diferencia de lo que ocurría con el obelisco o la pirámide, nos ofrece ya curvas animadas. Tenemos, pues, "formas orgánicas universalizadas".

\section{B. La religión del arte}

La religión del arte es el saber de sí del espíritu moral, pues el espíritu absoluto se manifiesta en el arte. Este espíritu real o espíritu verdadero (espíritu objetivo) ya no es el espíritu del despotismo oriental 
o de los poblados insociables inmersos todavía en la vida de la naturaleza, sino que es el espíritu sustancial de una ciudad humana que ha superado el estado salvaje de la naturaleza y que todavía no ha alcanzado la abstracción y el dolor de la subjetividad. Es el momento de la "bella individualidad". La ciudad aparece como una obra consciente de sí, como "un espíritu universal individualizado y concreto" (Hyppolite, 495).

La belleza del arte antiguo aparece cuando el espíritu se ha elevado por encima de su realidad, cuando ha retornado de su verdad objetiva al puro saber de sí mismo. Así, pues, el arte deja de ser el ser moral para convertirse en la reminiscencia e interiorización de dicho ser. Para Hegel, "esta conciencia es el espíritu cierto dentro de sí, que se duele de la pérdida de su mundo y que ahora hace surgir su esencia, elevada por sobre la realidad, desde la pureza del sí mismo".

En efecto, cuando el espíritu griego se convierte en saber de sí mismo y se reproduce en la obra de arte, esta reminiscencia es el signo de una forma más elevada. La evolución de la religión del arte es su tránsito a la subjetividad abstracta, al puro concepto, que en la creación artística todavía no es más que la forma de la actividad que crea. Para Hegel, "esta forma es la noche en que la sustancia es traicionada y se ha convertido en sujeto; de esta noche de la certeza pura de sí mismo resurge el espíritu moral como la figura liberada de la naturaleza y de su ser allí inmediato" (Hegel, 409).

En efecto, esta noche es la actividad creadora del sujeto que desaparece en primer lugar en su obra y todavía no se representa como tal a sí mismo. De ahí que Hegel señale que "esta pura actividad, consciente de su fuerza inalienable, lucha con la esencia no configurada (su pathos); haciéndose dueña de ella, ha convertido el pathos en su materia y se ha dado su contenido; y esta unidad surge como obra, como el espíritu universal individualizado y representado" (Hegel, 410).

Valls Plana, después de afirmar que este párrafo es de una belleza especial, donde Hegel nos da la clave de todo el arte griego, comenta que aquí el artista es sojuzgado por la fuerza universal de lo absoluto, sufre en su interior el pathos de la divinidad y pierde así la libertad de su autoconciencia. Pero esta violencia absoluta que padece el alma del artista es vencida a su vez por una fuerza igualmente absoluta: "el puro sí mismo del individuo" (Valls Plana, 337).

Así, todo lo que puede ser representado objetivamente lo será en esta obra de arte divina. Todo, excepto el concepto, la absoluta certeza del sí mismo en el mismo. El espíritu griego es el espíritu que se manifiesta al exterior, pero que solo conoce del espíritu esta manifestabilidad.

Hegel anticipa al arte griego como primer grado del espíritu absoluto, ya que el arte egipcio, estudiado dentro de la sección dedicada a la religión natural, no es el arte auténtico porque es "instintivo", y aunque busca el pensamiento alcanza solo la abstracción. En efecto, el arte griego brota ya de una autoconciencia que se reconoce como tal, es ya consciente de la absolutez de lo humano y lo representa por ello como divino.

De ahí que la religión revelada sea considerada luego como un estadio superior del espíritu en el cual ya no se da tampoco arte propiamente dicho. En la religión cristiana lo representado se identificará con la representación misma en virtud de la Encarnación: Jesucristo (Dios y hombre a la vez) no es ya una 
representación de la divinidad, sino que es Dios mismo, y en ese sentido las representaciones religiosas cristianas dejan de ser meramente artísticas.

Al respecto, Hyppolitte señala que en la religión cristiana la revelación es completa, pero tal revelación es más que la manifestación objetiva; lleva en sí el momento de la negación de esta manifestación, lo profundo de la noche, mientras que el espíritu artista solo conoce la vida del día. De este modo, la religión del arte, en su dialéctica, nos abre el camino hacia la revelación de la subjetividad, a la cual se eleva ya en sí en este saber de su sustancia. "Esta absoluta certeza del sî mismo en que se ha disuelto la sustancia debe convertirse finalmente en la figura en la cual se contempla el espíritu. Entonces la tragedia antigua, que todavía no es más que representación, se habrá convertido en drama cristiano, el drama de la vida y de la muerte de un Dios que se realiza efectivamente en la historia. Así pues, esta dialéctica va de la obra objetiva y trascendente a la subjetividad, de la naturaleza al espíritu, de la coseidad al sí mismo, de la sustancia al sujeto. Los tres momentos de este devenir son: la obra de arte abstracta, donde el espíritu ético se aparece en la forma de puras figuras divinas; la obra de arte viviente, donde el hombre pasa a ser la figura elaborada de lo divino en las fiestas y los juegos; y la obra de arte espiritual, donde el espíritu reside en el lenguaje de la epopeya, de la tragedia y de la comedia. Este lenguaje reproduce en su evolución el devenir de la sustancia devolviéndose en la pura certeza de sí. La epopeya reproduce la figura plástica de los dioses, la tragedia continúa la obra de arte viviente; finalmente en la comedia, el hombre se sabe como destino de los dioses; absorbe en él toda la sustancialidad, es la conciencia dichosa, pero es también la conciencia desgraciada que todavía se ignora" (Hyppolite, 427).

\section{Conclusión}

La religión natural y del arte son presentadas por Hegel como una anticipación, no sólo de la religión revelada sino también de la misma filosofía, pues se trata del despliegue del espíritu absoluto dentro de la lógica de la representatividad, que aún no es la del concepto, y puesto que el desarrollo de la conciencia o espíritu subjetivo (la fenomenología), -que está en permanente interacción con el mundo que nos rodea (espíritu objetivo)-, aún no alcanza con ellas su más alto desarrollo (espíritu absoluto), la unidad del en sí y para sí.

Asimismo, las configuraciones de la religión que hemos presentado se han ordenado de acuerdo con las configuraciones teleológicas de su objeto. Y en cada configuración la religión tiene un determinado contenido de verdad, sin ser, sin embargo, "la verdadera" religión". En este sentido Hegel hace justicia a las religiones no reveladas presentando la verdad relativa y no absoluta de las mismas.

Finalmente, Hegel logra diferenciar tres intuiciones asociadas a tres tipos de religión: la intuición de Dios en la forma de la naturaleza, la intuición de Dios en las obras de arte, y la intuición de Dios en la verdadera forma originaria del espíritu. Son grados que se corresponden respectivamente a la religión natural, a la del arte y a la revelada; en donde la primera, el espíritu es en la forma de la conciencia; en la segunda, en la de la autoconciencia y; en la tercera, en la forma de la unidad de ambas. Es el proceso del espíritu que va desde la certeza sensible hasta el entendimiento, pasando por la percepción, con el fin de alcanzar la universalidad abstracta de la representación, para llegar al final al concepto especulativo. 


\section{REFERENCIAS}

Hegel, G.W.F. (1986) Fenomenología del Espíritu. Traducción de Wenceslao Roces, con la colaboración de Ricardo Guerra. México: FCE.

Hegel, G.W.F. (1984) Lecciones sobre filosofía de la religión. Traducción de Ricardo Ferrara. Buenos Aires-Madrid: Alianza Editorial.
Hyppolite, J. (1974) Génesis y estructura de la fenomenología del espiritu de Hegel. Barcelona: Península.

Valls Plana, R. (1994) Del yo al Nosotros. Lectura de la Fenomenología del espíritu de Hegel. Barcelona: PPU. 